\title{
Applying remote health monitoring to understand users' QoE in multisensory applications in real-time
}

\author{
JORDANO R. CELESTRINI, Federal University of Espirito Santo, Brazil \\ ESTÊVÃO B. SALEME, Federal Institute of Espirito Santo, Brazil \\ NIALL MURRAY, Athlone Institute of Technology, Ireland \\ RODRIGO V. ANDREÃO, Federal Institute of Espirito Santo, Brazil \\ CELSO A. S. SANTOS, Federal University of Espirito Santo, Brazil
}

User's Quality of Experience (QoE) understanding from objective metrics has been increasingly explored in multisensory research. However, capturing physiological data adds a degree of difficulty to an already complex environment composed of software to reproduce content and actuators to deliver sensory effects. In this paper, we introduce the potential use of remote patient monitoring (RPM) systems to monitor users' QoE through a specific tool named HealthDash. We aim to raise discussion around them in digital multisensory experiences, their application, advantages and disadvantages, and challenges and opportunities.

CCS Concepts: • Hardware $\rightarrow$ Sensor applications and deployments; • Applied computing $\rightarrow$ Health informatics; • Human-centered computing $\rightarrow$ HCI design and evaluation methods; Virtual reality.

Additional Key Words and Phrases: IoT, multisensory, user monitoring, health sensors

\section{ACM Reference Format:}

Jordano R. Celestrini, Estêvão B. Saleme, Niall Murray, Rodrigo V. Andreão, and Celso A. S. Santos. 2021. Applying remote health monitoring to understand users' QoE in multisensory applications in real-time. In SensoryX '21: Workshop on Multisensory Experiences, together with IMX 2021: ACM International Conference on Interactive Media Experiences. June 21-23, 2021. NY, USA. 4 pages.

\section{INTRODUCTION}

A trendy research topic in multimedia is how to understand users' QoE from objective metrics $[2,3,7,8]$. This involves capturing physiological data from users to obtain concealed data behind subjective experiments. Cameras, eye-tracking devices, heart-rate monitoring wrists, and electroencephalography headsets are examples of equipment to this end. However, integrating them into users QoE experiments is not straightforward. If they provide an SDK or API, researchers and developers struggle to adapt them to their system; if data is captured separately by third-party software not integrated into the mulsemedia system, researchers have to synchronize them manually when the experience starts and finishes. Both of them take time, which could be spent on research instead.

Remote patient monitoring (RPM) is a strategy to improve patient care using digitally transmitted health-related data, captured by intelligent sensors, wearable or hand-held devices, mobile phones, and implanted monitoring devices [4]. RPM systems can be a useful alternative to monitor QoE experiments, as they allow physiological parameters to be monitored continuously using devices or sensors. A proposal that integrates sensors to capture users' physiological data automatically in XR and multimedia systems would save not only researchers time, but also organize data in a

Published in accordance with the terms of the Creative Commons Attribution 4.0 International Public License (CC BY 4.0). Permission to reproduce or distribute this work, in part or in whole, verbatim, adapted, or remixed, is granted without fee, provided that the appropriate credits are given to the original work, not implying any endorsement by the authors or by SBC.

(C) 2021 Brazilian Computing Society 
fashion that is ready to be analyzed. This could potentially boost objective metrics in mulsemedia experiments and, as a result, grow more interest in developing QoE models from objective measures.

Many solutions have been developed focusing on the RPM context. Su et al. [11] proposed an IoT framework for RPM systems to aging people, capable of communicating with sensors and automatically triggers medical alerts. In the same direction, Hamim et al. [6] created an RPM system for patients and aging people. The solution is composed of hardware to monitor heart rate and galvanic skin responses, and also by an Android application, where the data captured from patients can be visualized. Fazio et al. [5] proposed the use of the FIWARE cloud platform to accelerate the development of RPM solutions.

In this paper, we propose the use of HealthDash [1] - a remote patient monitoring platform - for gathering users' physiological data during multisensory QoE experiments performing. We claim that this novel approach towards continuously monitor users' QoE (through cameras, eyetracking devices, physiological signals capture) would facilitate a deep understanding of users' physiological state during digital multisensory experiences, which spark many different human sensations. The remainder of the paper is organized as follows: Section II presents HealthDash RPM Platform and details how it can be used to monitor users in QoE experiments, and Section III brings a discussion about challenges regarding this integration and concludes with some opportunities for future work.

\section{HEALTHDASH FOR CONTINUOUS QOE MONITORING}

HealthDash is an RPM platform that enables monitoring users' state through sensors continuously, obtaining physiological data and synchronizing them to a cloud application, where the data can be processed. A typical architecture of an RPM platform is depicted in Figure 1. In this approach, data is collected from the patient (or user) through health devices and synchronized to the cloud using a gateway, which is an embedded software capable of communicating with health devices and transmitting data over the Internet. The gateway can perform other tasks, including data compression, local storage, data mining, and security-related tasks.

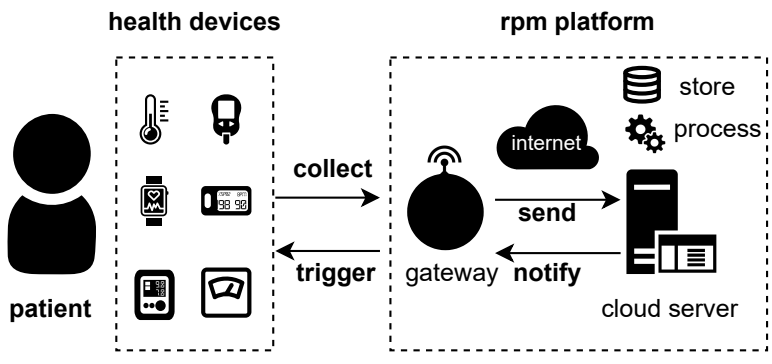

Fig. 1. RPM typical architecture - data is collected from users (patient) stored and processed in the cloud and may trigger events.

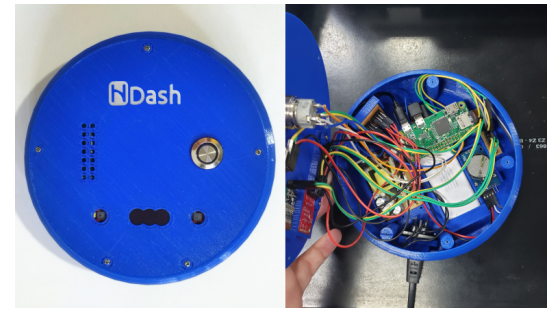

Fig. 2. HealthDash autonomous gateway - a bespoke hardware and embedded software.

Two types of gateways are provided by HealthDash to perform these tasks: an autonomous one, which allows monitoring without any interaction with the user, and an interdependent one, which requires at least some degree of user interaction. The interdependent gateway usually takes advantage of a device that the user already has, such as a mobile phone, tablet, or even a TV. In this case, HealthDash provides the software to the gateway, leaving to the user the responsibility to configure the devices and synchronize the data. On the other hand, the autonomous gateway is usually used in controlled environments, where it is possible to perform a pre-configuration of the devices. For this type of gateway, in addition to providing the necessary software, HealthDash also provides a hardware architecture to run the application, as shown in Figure 2. 
The reference hardware of the HealthDash autonomous gateway uses a RaspberryPI Zero W, a single-board computer with support for Wi-Fi and Bluetooth, making it ideal for communicating with health devices and also to synchronize data with the HealthDash Cloud. Moreover, this version of the gateway supports the connection of sensors for environment monitoring, allowing the acquisition of contextual information during the experiments' execution. The software that runs on the HealthDash Gateway is developed in Javascript, allowing it to be executed on a variety of operating systems. This feature makes it possible to use the same reference implementation to execute HealthDash on both the autonomous and the interdependent gateway.

For continuous QoE monitoring, HealthDash presents itself as an alternative for capturing and storing data, since it already provides all the hardware and software infrastructure necessary for monitoring users. Furthermore, the cloud application provided by the platform also has integration APIs, through which it is possible to access the information captured by the sensors and correlate the data with other sources of information. For digital multisensory experiences, we suggest it could be used along with a mulsemedia platform, such as PlaySEM Video Player and PlaySEM SER 2 [9], to provide an end-to-end ecosystem that allows audiovisual and sensory effects rendering and physiological data analysis for QoE assessment.

In our approach, HealthDash would be responsible for the acquisition of physiological data from users whilst PlaySEM would take care of the mulsemedia part of the experiments. In HealthDash, data is stored according to a predefined scheme, which can be managed through an administrative interface. The data schema uses the JSON Schema Specification and defines the structure of the data to be stored. This allows defining the physiological parameters according to the experiment that will be performed - mapping them to their respective schemes - which will be captured and stored in the HealthDash database.

\section{CONCLUDING DISCUSSION}

Recent work has explored the potential of physiological data collected from health devices for recognition of emotions, mainly in areas such as human-computer interaction, software engineering and health. Brain signals, galvanic skin response, electrical activity produced by skeletal muscles, heart rate, blood volume and breathing metrics are parameters commonly used for this purpose. For the assessment of QoE, we understand that the recognition of emotions based on physiological data is a crucial tool for objectively quantifying human behavior [10]. However, capturing this information from health devices is still a challenge, given the heterogeneity of the devices employed, both in terms of connectivity protocols (USB, WiFi, Bluetooth, etc.), and in terms of communication protocols. A platform that promotes the integration of new devices, like HealthDash, might help speed up the development of solutions for QoE assessment, eventually boosting and fostering research in this area.

The outcome of HealthDash could be applied, for instance, to virtual reality applications used for training purposes. These physiological data might be used to interpret users' efficiency, attention span, health state, among other parameters, in work simulations avoiding foreseeable incidents and improving productive aspects. HealthDash, however, like other RPM systems, is time-based and it stores the date and time when some information was gathered from the user. Thus, when it comes to event-based applications (such as games and virtual reality applications) RPM systems are not fully ready in terms of the time an event was triggered - a synchronisation clock must exist between the application and the RPM system to record the exact moment an event happened and the time the physiological data were captured. This has to be taken into account when designing multisensory experiments using RPM systems to monitor QoE. Likewise, there is an opportunity for Artificial Intelligence tools to be integrated into this context to explore the data collected and 
stored by HealthDash. This would provide insightful tips on patterns found in the data, suggesting users' perception from objective metrics in real-time.

Future work includes experiments with users to validate the integration between HealthDash and PlaySEM, using the integration proposal presented in this work as an objective means to evaluate the user experience during multisensory QoE experiments. In addition to the user's physiological data, it is planned to adapt HealthDash to gather information from the environment, making it possible to correlate it with the experience reported by the user during the execution of the experiments.

\section{ACKNOWLEDGMENTS}

This study is partially supported by the National Council for Scientific and Technological Development (CNPQ, Brazil) and by the Espírito Santo Research and Innovation Support Foundation (FAPES, Brazil).

\section{REFERENCES}

[1] J. R. Celestrini, A. M. Baldi, R. V. Andreão, J. G. Pereira-Filho, and C. A. S. Santos. 2021. Flow-Based Situation-Aware Approach for eHealth Data Processing. In 2020 IEEE International Conference on E-health Networking, Application Services (HEALTHCOM). 1-7. https://doi.org/10.1109/HEALTHCOM49281.2021.9398992

[2] A. Covaci, E. B. Saleme, G. Mesfin, N. Hussain, E. Kani-Zabihi, and G. Ghinea. 2020. How Do We Experience Crossmodal Correspondent Mulsemedia Content? IEEE Transactions on Multimedia 22, 5 (2020), 1249-1258. https: //doi.org/10.1109/TMM.2019.2941274

[3] D. Egan, S. Brennan, J. Barrett, Y. Qiao, C. Timmerer, and N. Murray. 2016. An evaluation of Heart Rate and ElectroDermal Activity as an objective QoE evaluation method for immersive virtual reality environments. In 2016 Eighth International Conference on Quality of Multimedia Experience (QoMEX). 1-6. https://doi.org/10.1109/QoMEX. 2016.7498964

[4] F. A. C. Farias, M. Dagostini, C, Y. A. Bicca, V. F. Falavigna, and A. Falavigna. 2020. Remote Patient Monitoring: A Systematic Review. Telemedicine and e-Health 26, 5 (2020), 576-583. https://doi.org/10.1089/tmj.2019.0066 PMID: 31314689.

[5] M. Fazio, A. Celesti, F. G. Márquez, A. Glikson, and M. Villari. 2015. Exploiting the FIWARE cloud platform to develop a remote patient monitoring system. In 2015 IEEE Symposium on Computers and Communication (ISCC). 264-270. https://doi.org/10.1109/ISCC.2015.7405526

[6] M. Hamim, S. Paul, S. I. Hoque, M. N. Rahman, and I. Baqee. 2019. IoT Based Remote Health Monitoring System for Patients and Elderly People. In 2019 International Conference on Robotics,Electrical and Signal Processing Techniques (ICREST). 533-538. https://doi.org/10.1109/ICREST.2019.8644514

[7] C. Keighrey, R. Flynn, S. Murray, S. Brennan, and N. Murray. 2017. Comparing User QoE via Physiological and Interaction Measurements of Immersive AR and VR Speech and Language Therapy Applications. In Proceedings of the on Thematic Workshops of ACM Multimedia 2017 (Mountain View, California, USA) (Thematic Workshops '17). Association for Computing Machinery, New York, NY, USA, 485-492. https://doi.org/10.1145/3126686.3126747

[8] G. Mesfin, E. B. Saleme, O. A. Ademoye, E. Kani-Zabihi, C. A. S. Santos, and G. Ghinea. 2021. Less is (Just as Good as) More - an Investigation of Odor Intensity and Hedonic Valence in Mulsemedia QoE using Heart Rate and Eye Tracking. IEEE Transactions on Multimedia 23 (2021), 1095-1105. https://doi.org/10.1109/TMM.2020.2992948

[9] E. B. Saleme, C. A. S. Santos, and G. Ghinea. 2019. A mulsemedia framework for delivering sensory effects to heterogeneous systems. Multimedia Systems 25, 4 (01 Aug 2019), 421-447. https://doi.org/10.1007/s00530-019-00618-8

[10] D. P. Salgado, T. B. Rodrigues, C. Keighrey, R. Flynn, E. L. M. Naves, N. Murray, and F. R. Martins. 2018. A QoE assessment method based on EDA, heart rate and EEG of a virtual reality assistive technology system. Proceedings of the 9th ACM Multimedia Systems Conference, MMSys 2018 18, 517-520. https://doi.org/10.1145/3204949.3208118

[11] C. Su, J. Hajiyev, C. J. Fu, K. C. Kao, C. H. Chang, and C. T. Chang. 2019. A novel framework for a remote patient monitoring (RPM) system with abnormality detection. Health Policy and Technology 8, 2 (2019), 157-170. https: //doi.org/10.1016/j.hlpt.2019.05.008 